\title{
Physical Guidance of Cultured Retinal Neurons Using Zig-zag Surface Patterns
}

\author{
Saba Moslehi ${ }^{1,2}$, William J Watterson ${ }^{1,2}$, Conor Rowland ${ }^{1,2}$, Julian H. Smith ${ }^{1,2}$, Maria-Thereza \\ Perez $^{3,4}$ and Richard P Taylor ${ }^{1,2 *}$ \\ ${ }^{1}$ Physics Department, University of Oregon, USA
}

${ }^{2}$ Material Science Institute, University of Oregon, USA

${ }^{3}$ Department of Clinical Sciences Lund, Division of Ophthalmology, Lund University, Sweden

${ }^{4}$ NanoLund, Lund University, Sweden

*Corresponding author: All authors equally contributed

Richard Taylor, Physics Department, University of Oregon, Eugene, OR, 97403, USA

Saba Moslehi, Physics Department, University of Oregon, Eugene, OR, 97403, USA,

Maria Thereza Perez, Department of Clinical Sciences Lund, Division of Ophthalmology, Lund University, SE-221 85 Lund, Sweden

To Cite This Article: Saba M, William J W, Conor R, Julian H. S, Maria-Thereza P, Richard P T. Physical Guidance of Cultured Retinal Neurons Using Zig-zag Surface Patterns. Am J Biomed Sci \& Res. 2020 - 11(3). AJBSR.MS.ID.001629. DOI: 10.34297/AJBSR.2020.11.001629.

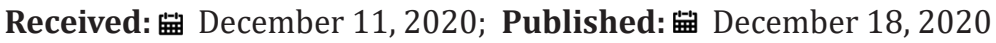

Keywords: Fractal, In Vitro, Physical Cues, Retinal Neurons, Su8

\section{Introduction}

The use of physical cues to control and guide various types of cells in vitro, especially neurons and their processes, has been the focus of a large amount of research. The response of neuronal processes to artificial surfaces depends on a number of factors including the cell type, the surface chemistry of the material, and the surface's topological features [1,2]. In this Opinion piece, we investigate the extent to which retinal neuronal processes can be made to follow straight lines patterned into a surface. We show they can follow lines with relatively shallow heights of $2 \mu \mathrm{m}$ and be made to undergo directional changes as great as $50^{\circ}$. However, some processes leave the lines and assume a weaving trajectory as they grow into the surface's unpatterned regions. Based on these findings, we propose that neuronal processes will follow lines more closely if their shapes mimic the fractal weave patterns of unrestricted neurons. In addition to exploring the fundamental behavior of neurons interacting with artificial surfaces, the results inform the design of bio-inspired electrodes for human implants.

\section{Materials and Methods}

Silicon substrates were cleaned using a 1-minute soak in sulfuric acid at $80^{\circ} \mathrm{C}$ followed by a 1-minute soak in deionized (DI) water at room temperature. The substrates were then exposed to oxygen plasma in a March CS-1701 Plasma Cleaner for 1 minute at $50 \mathrm{~W}$ and 70 mTorr. A $2 \mu \mathrm{m}$ thick layer of SU8 negative photoresist was then spin coated on the substrates. A ZEISS Ultra-55 Scanning Electron Microscope was used for e-beam lithography. A $5 \times 5 \mathrm{~mm}^{2}$ area of the substrate was patterned with parallel rows of SU8 zigzag lines. Because the SU8 was removed in the spaces between the lines, these regions had a Si surface. The height and width of the lines were both $2 \mu \mathrm{m}$. The angles of the zig-zag lines were $50^{\circ}, 70^{\circ}$, $90^{\circ}$ and $150^{\circ}$ (constant for each substrate). The substrates were modified by immobilizing laminin following protocols explained elsewhere to increase the surface biocompatibility prior to the cell cultures [3]. The substrates were then covered with a suspension of dissociated mouse retinal cells, obtained following ethical approval 
and protocols previously described elsewhere [4]. Neuronal and glial cells were identified using immunofluorescence.

\section{Results}

Qualitative observations were made after 3 and 7 days in vitro (DIV). At 3 DIV, processes emanating from cell bodies in close contact with the SU8 lines, tended, in most cases, to follow the lines. In some cases, the processes even made directional changes when reaching the line's turning points (Figure 1a-1d). However, some processes did leave the lines both in the straight sections (Figure 1b) and at their turning points (Figure 1c). The processes of neurons located away from the lines did not follow straight trajectories and instead weaved across the 2-D surface (Figure 1d). Whereas glial cells did not yet appear for these shorter culture times, they covered both the patterned and unpatterned regions of the substrate at 7 DIV. Although the glia rarely responded to the lines, occasionally the lines seemed to restrict their growth. Furthermore, a few neuronal processes followed the glial fibers rather than the lines at these later times (Figure 1e,f).
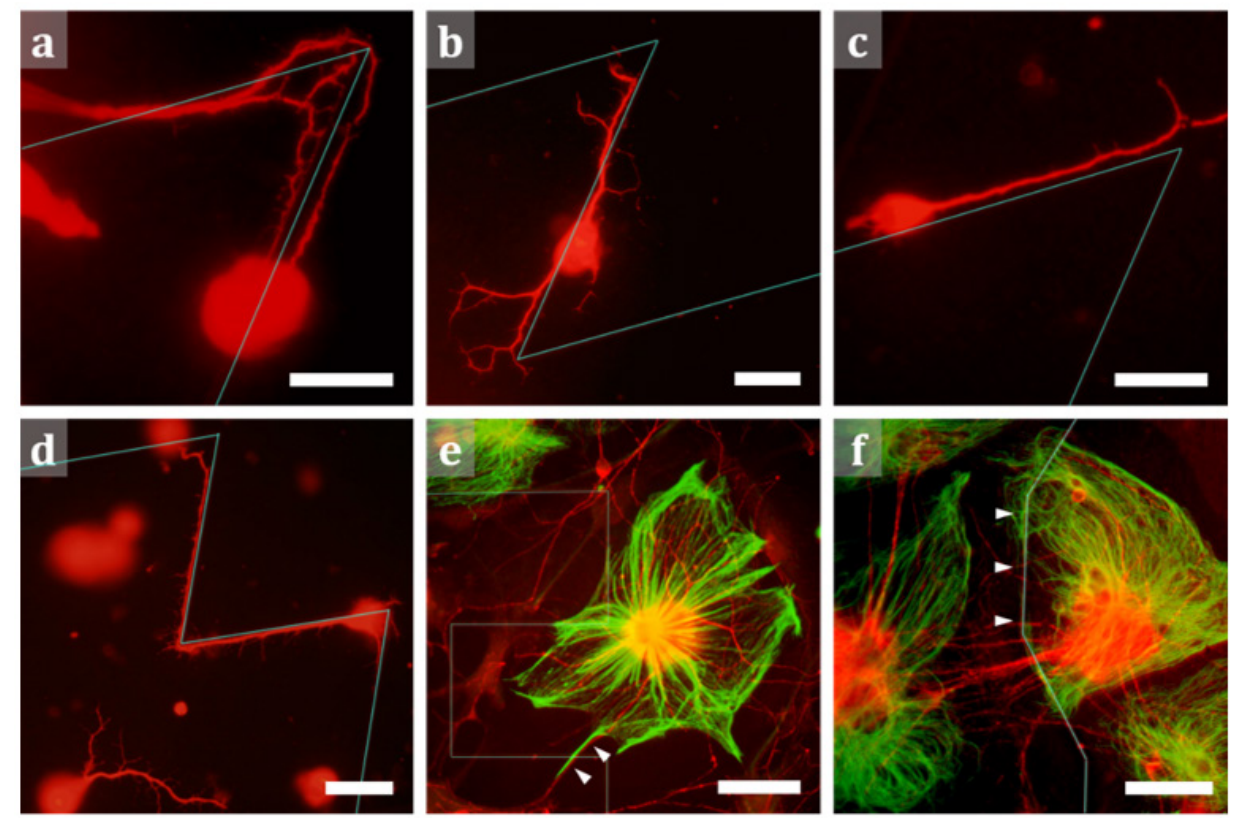

Figure 1: Fluorescence images of retinal cells interacting with SU8 lines. (a) Neuronal processes observed making a $50^{\circ}$ turn to follow the line pattern instead of leaving it for the smooth, unpatterned surface. (b) Neuronal processes observed following the line of a $50^{\circ}$ angled pattern. After branching, some processes leave the line. (c) A neuronal process observed following the line of a $50^{\circ}$ pattern; processes then leave the line at its turning point. (d) Neuronal processes observed growing on the nearby unpatterned surface show morphological differences to processes following a $70^{\circ}$ angled pattern. (e) The combined fluorescence image of neurons (red) and glia (green) on the surface of a $90^{\circ}$ pattern; the presence of glial cells affects the neuronal process behaviour; although near the pattern, the processes do not follow the lines, but they can, instead be seen to follow a glial process (arrowheads). (f) The combined fluorescence image of neurons (red) and glia (green) on the surface of a $150^{\circ}$ pattern; the left edge of the glial cell at the image center follows a segment of the line (arrowheads). Scale bars are $20 \mu \mathrm{m}$ in (a), (b), (c), (d) and $50 \mu \mathrm{m}$ in (e) and (f). The cyan lines mark where the zig-zag lines are located on the substrate surface. Panels (a-d) are images at 3DIV; panels (e, f) are at 7DIV.

\section{Discussion}

Neurons scan their environment for cues using motile structures called growth cones located at the tip of their processes. These structures have finger-like protrusions called filopodia containing F-actin proteins that are responsible for navigation and pathfinding in the 3-D environment [5]. When encountering 10 $\mu \mathrm{m}$ tall steps (which approximately match the size of the in-plane width of growth cones), it has been shown that typically half of the neuronal processes ignore the topographical cues [6]. Intriguingly, the height of our lines is much smaller than the typical growth cone size and yet retinal neuronal processes were still able to detect and follow their cues, similar to our previous observations using $4 \mu \mathrm{m}$ tall gallium phosphide nanowires [7]. In this study, we show that the processes occasionally even follow the lines when they changed direction. We hypothesize that there will be a minimum angle that neuronal processes can turn through based on their tolerance for bending-induced strains on their cytoskeleton. This hypothesis is based on previous findings that neuronal processes have the tendency to follow the direction of minimum principal curvature [8].

The presence of glial cells seemed to prevent neuronal processes from detecting the lines in most cases. Whereas previous research shows that, in vitro, glial cells adhere strongly to rigid substrates such as Si [9], the neurons prefer to adhere to and grow more processes on soft substrates [10]. Given that glial cells are twice as soft as neurons [11], they are free to grow in the areas between the lines, extending processes in all directions. As these cells secrete 
several neurotrophic and neurite guiding factors [12], neuronal processes also tend to abound in the vicinity of glial processes and, as seen here, tend also to occasionally follow a glial process. It is, thus, reasonable to assume that chemical cues provided by the glial cells are stronger than the topographic cues provided by the lines.

\section{Conclusion}

While the role of physical and chemical cues as a means to engineer and guide cell behavior is well-established, more attention needs to be paid to the natural behavior of neurons when patterning physical cues. The preliminary results shown in this Opinion, highlight the difference in neuronal morphology between the guided and the unrestricted behavior of neuronal processes. Although processes can be made to follow straight lines, and even in some instances turn through sharp corners to do so, a number of processes nevertheless leave these artificial patterns. When they do so, they assume the morphology observed for unrestricted neuronal processes on the smooth, unpatterned surfaces. Recent studies have shown that neurons follow fractal weave patterns when connecting to their neighbors in the body's neural network [13]. We propose that the neuronal processes observed leaving our lines are reverting to this natural behavior. By designing physical cues composed of fractal branches that optimize branching and weaving angles that mimic this natural behavior, processes might follow these cues more closely. If shown, this novel 'fractal resonance' between neurons and artificial lines could be exploited for stimulating/recording electrodes that interface with neural tissue. Such designs would need to accommodate the presence of other cell types, such as glial cells, and their effects on process outgrowth.

\section{Acknowledgements}

RPT is a Cottrell Scholar of the Research Council for Science Advancement. This research is supported by the WM Keck Foundation (RPT) and The Swedish Research Council (M.-T.P.: 2016-03757), Crown Princess Margareta's Committee for the Blind, Stiftelse för Synskadade i fd Malmöhus Län and the Crafoordska Stiftelsen.

\section{Conflict of interest}

There is no conflict of interest.

\section{References}

1. Hoffman-Kim D, Mitchel JA, Bellamkonda RV, (2010) Topography, Cell Response, and Nerve Regeneration. Annu. Rev Biomed Eng 12: 203-231.

2. Rajnicek A, Britland S, McCaig C (1997) Contact guidance of CNS neurites on grooved quartz: influence of groove dimensions, neuronal age and cell type. J Cell Sci 110: 2905-2913.

3. Xue P, Bao J, Chuah Y J, Menon N V, Zhang Y, et al. (2014) Protein Covalently Conjugated SU-8 Surface for the Enhancement of Mesenchymal Stem Cell Adhesion and Proliferation. Langmuir 30: 3110-3117.

4. Watterson W J, Moslehi S, Rowland C, Zappitelli K M, Smith J H, et al. (2020) The Roles of an Aluminum Underlayer in the Biocompatibility and Mechanical Integrity of Vertically Aligned Carbon Nanotubes for Interfacing with Retinal Neurons. Micromachines 11(6): 546.

5. Mattila P K, Lappalainen P (2008) Filopodia: molecular architecture and cellular functions. Nat Rev Mol Cell Biol 9: 446-454.

6. Li N, Folch A (2005) Integration of topographical and biochemical cues by axons during growth on microfabricated 3-D substrates. Exp Cell Res 311: 307-316.

7. Piret G, Perez MT, Prinz C N (2015) Support of Neuronal Growth Over Glial Growth and Guidance of Optic Nerve Axons by Vertical Nanowire Arrays. ACS Appl Mater Interfaces 7: 18944-18948.

8. Smeal R M, Rabbitt R, Biran R, Tresco P A, (2005) Substrate Curvature Influences the Direction of Nerve Outgrowth. Ann Biomed Eng 33: 376382.

9. Georges PC, Miller WJ, Meaney DF, Sawyer ES, Janmey PA (2006) Matrices with Compliance Comparable to that of Brain Tissue Select Neuronal over Glial Growth in Mixed Cortical Cultures. Biophys J 90: 3012-3018.

10. Georges PC, Miller WJ, Meaney DF, Sawyer ES, Janmey PA, (2006) Neurite branching on deformable substrates. Neuroreport 13: 2411-2415.

11. Lu Y B, Franze K, Seifert G, Steinhäuser C, Kirchhoff F, et al. (2006) Viscoelastic properties of individual glial cells and neurons in the CNS. Proc Natl Acad Sci USA 103: 17759-17764.

12. Rigby MJ, Gomez TM, Puglielli L (2020) Glial Cell-Axonal Growth Cone Interactions in Neurodevelopment and Regeneration. Front Neurosci 14.

13. Smith J H, Rowland C, Harland B, Moslehi S, Montgomery RD, et al. (2020) How Neurons Exploit Fractal Geometry to Optimize their Network Connectivity. Sci Rep. 